Table 3 Important reactions for differentiation of stored lipids in MLD and various lipoidoses

\begin{tabular}{l|c|c|c|c|c} 
& Tay-Sachs & Gargoylism & Gaucher & Niemann-Pick & MLD \\
\hline Sudan III & + & + & + & $+\sim++$ & \pm \\
Nile blue sulfate & ++ & ++ & ++ & ++ & ++ \\
PAS & +++ & ++ & +++ & $+\sim++$ & ++ \\
Smith-Dietrich & + & + & - & + & $-\sim+$ \\
Cerebroside & ++ & ++ & ++ & - & ++ \\
Phosphatide & + & ++ & - & ++ & $-\sim+$ \\
Acetic cresylviolett & ++ & - & \pm & - & +++ \\
Neutral red, pH under 1.5 & - & - & - & - & +++ \\
\multicolumn{1}{c|}{ pH 2 4 } & + & + & - & - & +++ \\
\hline
\end{tabular}

Summary

Histochemical studies were performed on the deposited substances in metachromatic leucodystrophy. The findings obtained correspond with the biochemical findings of increased sulfatide in this disease. And the acidic neutral red method was found to be effective for the diagnosis of metachromatic leucodystrophy.

\title{
References
}

1) Austin, J.H. : Proc. Soc. Exptl. Biol. Med., 100, 361-364, (1959).

2) Hazama, F., Suzuki, T. and Uesugi, Y.: Proc. Jap. Histochem. Assoc., 3, 84-90, (1962).

3) Jatzkewitz, H. : Hoppe-Seyler's Z. Physiol. Chem., 311, 279-282, (1958). Jap., 14, 413-433, (1954).

\section{On the Histochemical Study of the Various Organs of Spontaneously Hypertensive Rats}

\author{
Kozo Okamoto, Fumitada Hazama, Toshio Takeda, Ryo Tabei, \\ Shoichiro Nosaka, Masakazu Fukushima, Yukio Yamori, \\ Masao Matsumoto
}

(Director: Prof. Kozo OKAMOTO)

From among many rats of the Wistar strain, a spontaneously developed hypertensive male was selected. After persisting a hypertensive state for over a month, this rat was mated with a female having blood pressure slightly above the average to obain $F_{1}$ rats. Many of these $F_{1}$ rats showed spontaneous hypertension. The process of mating these rats was repeated successively. In these rats, $100 \%$ occurence of spontaneous hypertension in $F_{2}$ rats onward was 
observed within the first 5 months of life. These rats are referred to as spontaneously hypertensive rats" (S.H. rats).

These animals were sacrificed in the pre-hypertensive stage (40 to 60 days after birth), initial stage (5 to 8 months after birth) or advanced stage of hypertension (14 to 18 months after birth).

Twenty-one kinds of enzyme activity were examined histochemically in the adrenals, thyroid, kidneys, heart, liver and pancreas, and noradrenalin reaction was also applied to the adrenal medulla. Each positive finding in S.H. rats was obtained by comparing it with the reaction of the same sex normotensive rats with almost the same survival duration.

Fist, findings which were detected from the pre-hypertensive stage are presented.

1) Increase of G-6-PD activity in the adrenal cortex, especially in the middle and outer part of its fascicular zone. The enzyme, G-6-PD in the adrenal cortex is considered to have a function simlar to the TPNH generator. This TPNH is necessary as a cofactor for the hydroxylase which is important for steroid synthesis. Consequently, the elevation of the G-6-PD activity in the adrenal cortex seems to suggest the increase of steroid synthesis in the adrenal cortex of the S.H. rat.

2) Enhancement of TPND activity in the adrenal cortex. This means also the rise of oxidation of much of the TPNH throuh this enzyme. This finding should be thought of as an incidental change to the enhancement of the G6-PD activity.

3) Marked increase of norepinephrine contet in the adrenal medulla. By the noradrenalin reaction for the adrenal medulla, using both osmium and iodate reactions, the noradrenalin-storing cell islets in the adrenal medulla of S.H. rats indicate a stronger reaction with about twice the dimensional ratio to the whole adrenal medulla histometrically, than is found in normotensives. The appearance of this finding seems to depend on the increase of either production of noradrenalin in these reaction positive cells, or retention of noradrenalin because of the decrease of secretion. This latter is unaffected by the production rate of noradrenalin in these cells which is normal or below average. It cannot be decided at the present stage, which interpretation is more probable.

4) Elevation of ACPase activity in the follicular cells of the thyroid. This seems to suggest that there exists an increase of secretory activity of the thyroid in S.H. rats.

Second, the findings which can be seen after the incidence of hypertension are given.

5) Increase of ATPase activity in the wall of renal arterioles in the kidney. From this finding, it can be considered that the renal arterioles of S.H. rats tend to contract more intensely than those of normtensives in the hypertensive state.

6) Elevation of MAO activity in the heart muscle. Gradual increase of the activity was observed after the incidence of hypertension and dominant increase was detected in the advanced hypertensive stage. The higher the level 
of blood pressure, the stronger the observed reaction in the heart. This finding seems to indicate that the monoamine metabolism, primarily oxidation, in the heart muscle is markedly enhanced in the hypertensive condition.

Further, some enzyme histochemical studies were applied to the hypothalamus of S.H. rats and of the controls. The following results were obtained from the hypothalamus of the S.H. rats in the pre-hypertensive stage compared to the controls.

7) Constant elevation of ACPase activity was observed in nerve cells in the lateral preoptic, supraoptic, ventromedial, dorsomedial, arcuate, posterior hypothalamic nuclei and in some parts of the mamillary nuclear complex. Increase of DPND activity was observed in the cells of the supraoptic nucleus and both the DPNO and the MAO activities were found to be enhanced in parts of the ventromedial nucleus and some additional findings were also detected in these two enzyme activities. These findings are almost all localized in the lateral preoptic and postero-medial parts of the hypothalamus.

There is evidence to suggest that increase of ACPase activity in nerve cells is closely related to neural activity. Based on this, the following presumption seems to be applicable: In the S.H. rats, there exists from the pre-hypertensive stage, overactivity of both the lateral preoptic area (parasympathetic zone) and the postero-medial area (sympthetic zone) of the hypothalamus coincidentally. Further, the elevation of DPND activity in nerve cells is reported to suggest the enhancement of respiratory function of the cells. Considering this, the findings derived from investigations of DPND activity can be assumed to be consistent with those of ACPase activity research in the hypothalamus of the S.H. rats.

On the basis of all the above-mentioned histochemical findings of the various organs of the S.H. rat and, in addition, results obtained from various other experiments for this $\mathrm{rat}^{2,3}$, studies on the mechanism of hypertension in the spontaneously hypertensive rat are now in progress in our laboratory.

\section{References}

1) Okamoto, K. and Aoki, K. : Development of a strain of spontaneously hypertensive rats. Jap. Circul. J., 27, 282-293, $1963 . \quad$ 2) Aoki, K., Tankawa, H., Fujinami, T., Miyazaki, A. and Hashimoto, Y.: Pathological studies on the endocrine organs of the spontaneously hyper. tensive rats. Jap. Heart J., 4, 426-442, $1963 . \quad 3$ 3) Okamoto, K., Aoki, K., Nosaka, S. and Fukushima, M. : Cardiovascular diseases in the spontaneously hypertensive rat. Jap. Circul. J., 28, 943-952, 1964. 